\title{
Severely Atrophic Muscle Fibers with Nuclear Clumps Survive many Years in Permanently Denervated Human Muscle
}

\author{
Helmut Kern ${ }^{1}$, Ugo Carraro², Donatella Biral ${ }^{3}$, Nicoletta Adami ${ }^{2}$ and Sandra Zampieri ${ }^{*}, 4$ \\ ${ }^{1}$ Ludwig Boltzmann Institute of Electrostimulation and Physical Rehabilitation, Department of Physical Medicine, \\ Wilhelminenspital, A-1171 Vienna, Austria \\ ${ }^{2}$ Laboratory of Translational Myology of the Interdepartmental Research Center of Myology, Department of Biomedical \\ Science, University of Padova, I-35121 Padova, Italy \\ ${ }^{3}$ C.N.R. Institute of Neuroscience, Department of Biomedical Science, University of Padova, I-35121 Padova, Italy \\ ${ }^{4}$ Division of Rheumatology, Department of Clinical and Experimental Medicine, University of Padova, Padova, Italy
}

\begin{abstract}
After complete lumbar-ischiatic spinal cord injury (SCI) the lower motor neuron (LMN) denervated human muscle fibers lose completely the myofibrillar apparatus and the coil distribution of myonuclei that are relocated in groups (nuclear clumps) in the center of these "severely atrophic" muscle fibers. In our cohort of patients, the "severely atrophic" myofibers are frequent in muscle biopsies harvested three to six years after SCI. Up to two years of LMN denervation the muscle fibers with nuclear clumps are $2 \pm 5 \%$ (mean $\pm \mathrm{SD}$ ) of the total muscle fibers. The percentage increases to $27 \pm$ $9 \%$ between three and six years of denervation $(\mathrm{p}<0.001)$, and then abruptly decrease from the 6th year of LMN denervation onward, when fibrosis takes over to neurogenic muscle atrophy. Immunohistochemical analyses show that nuclear grouping occurs in both fast and slow muscle fibers. These results show that human muscle fibers survive permanent denervation much longer than generally accepted.
\end{abstract}

Keywords: Human muscle, long-standing denervation, myonuclear clumps, nuclear clumps, spinal cord injury.

\section{INTRODUCTION}

The permanent injury of lower motor neurons causes rapid atrophy of skeletal muscle fibers that mainly occurs during the first few months. In rodents three to seven months after denervation, muscle fibers undergo a stage of slow progressive atrophy that result in a consistent reduction (up to $90 \%$ ) of the muscle tissue. At this late stage, the denervated muscle contains still numerous severely atrophic muscle fibers, some of which had lost all the contractile proteins and the coil distribution of myonuclei, which are aggregated in the center of the muscle fiber (nuclear clumps). At the same time, adipocytes and collagen sheets fill some of the empty spaces of the muscle and finally fibrosis prevails [1-3].

In biopsies of quadripeps muscle harvested from human subjects who have experienced complete lower motor neuron (LMN) lesion (Conus Cauda Syndrome) we observed similar events, but in a more extended period of time [4]. Mild atrophy (that corresponds approximately to a decrease in muscle fiber size of the $50 \%$ ) appears in few weeks. It progresses during the first two years of denervation, while severe atrophy (a stage in which muscle fiber size decreases to $20-10 \%$ with respect to normal values) appears after twothree years, accompanied by a progressive degeneration of the muscle tissue $[4,5]$. This behaviour is not unique to

*Address correspondence to this author at the Interdepartmental Research Center of Myology, Viale G. Colombo 3, I-35121 Padova, Italy; Tel:+ 39 049 8276359; Fax: +39 049 8276040; E-mail: sanzamp@unipd.it humans, but it is a common feature in mammals larger than rodents $[6,7]$.

After the second year of persistent LMN denervation, muscle tissue from these patients is progressively enriched of very atrophic fibers, which are depleted of myofibrillar apparatus, and in longitudinal section present aggregations of tens of nuclei (Nuclear Clumps) separated by stretches of empty myoplasm. In transverse sections, these severely atrophic muscle fibers show three or more central nuclei and typically no contractile structures.

In the present study, our aim was to stage and quantify severely atrophic human muscle fibers presenting nuclear clumps in complete lower motor neuron denervated quadriceps muscle biopsies harvested from one to ten years after lumbar-ischiatic spinal cord injury (SCI).

\section{MATERIALS AND METHODOLOGY}

\section{Patients}

We collected muscle biopsies from quadriceps muscles of twenty-four patients who had experienced since one to ten years, traumatic spinal cord injury that caused complete lesion of the lumbar-ischiatic lower motor neurons (complete Conus Cauda Syndrome). Demographic and clinical data of the patients are described in [8]. All subjects enrolled in the study were volunteers who received and signed a detailed informed consent. Clinical and functional assessments, as well as follow-up and muscle biopsies, were performed at the Wilhelminenspital, Vienna (Austria). Complete denervation of quadriceps muscle was assessed by electrophysiological testing, i.e. by test electrical stimulation, needle electromyo- 
graphy, brain motor control assessment, transcranial and lumbosacral magnetic stimulation [8]. All applicable rules concerning the ethical use of human volunteers were followed during this study (Approval of Ethical Committee, Vienna, Austria: EK-02-068-0702).

\section{Hystological Analyses}

Light microscopy and morphometry of muscle biopsies were performed at the Interdepartmental Research Center of Myology, University of Padova (Italy), as described in [9]. In brief, after either freezing or fixing and embedding the specimens, cryosections (10 $\mu \mathrm{m}$ thickened) or thin section (1 $\mu \mathrm{m}$ thickened), were collected on glass slides and stained with hematoxylin-eosin (H\&E) or toluidine blue, respectively, using conventional techniques. All images were collected using a Zeiss microscope connected to a Leica DC $300 \mathrm{~F}$ camera under the same conditions that were used to acquire a reference ruler. Data are expressed as mean \pm SD. To detect significant differences between groups, we performed the two-tailed Student's $t$-test for unpaired observations. The significance level was set to $\mathrm{p}=0.05$.

\section{Immunohistochemical Analyses}

Unfixed muscle sections were labeled for 1 hour at room temperature using mouse monoclonal antibody directed against slow-type myosin heavy chain (MHCs) (Novocastra, Newcastle-upon-Tyne, U.K), 1:20 diluted in Tris-buffered saline (TBS). Sections were rinsed $3 \times 5 \mathrm{~min}$ in TBS, and then incubated for 1 hour at room temperature with FITC labeled conjugates directed against mouse IgG (Sigma-Aldrich, St. Louis, USA) 1:200 diluted in 10\% goat serum/TBS. Negative controls were performed by omitting the primary antibodies on samples. After washes, nuclei were counterstained for $5 \mathrm{~min}$ at room temperature with Hoechst 33258 (Sigma-Aldrich, St. Louis, USA), sections were coverslipped using mounting medium (Dako, Glostrup, Denmark) and observed under a Zeiss microscope.

\section{RESULTS}

Fig. (1) show H\&E stained cross sections of skeletal muscle biopsies harvested from normal (A) and denervated subjects at 0,8 (B), and 4,1 (C) years after spinal cord injury. Normal muscle (A) is characterized by well-packed myofibers of $58,3 \pm 11,6$ mean fiber diameter $(\mu \mathrm{m} \pm \mathrm{SD})$. Minimal interstitial connective tissue is present. One year after complete lower motor neuron lesion (B), the muscle is still characterized by variable mild atrophy with myofibers of 30,2 $\pm 10,8$ mean fiber diameter $(\mu \mathrm{m} \pm \mathrm{SD})$. Noteworthy, nuclei are still peripherally located.

After four years from lower motor neuron injury $(\mathbf{C})$, muscle biopsy is characterized by numerous very atrophic fibers $(<15 \mu \mathrm{m}$ mean fiber diameter) separated by increased fibrous tissue. Arrowheads point to the severely atrophic myofibers in which the contractile apparatus seems to be almost absent and five or more nuclei fill the cross section of some of them (nuclear clumps).

Table 1 show the percentage of severely atrophic muscle fibers with nuclear clumps (nuclear clumps/total muscle fibers $x$ 100) in muscles of denervated patients listed according their time of $\mathrm{LMN}$ denervation. Severely atrophic
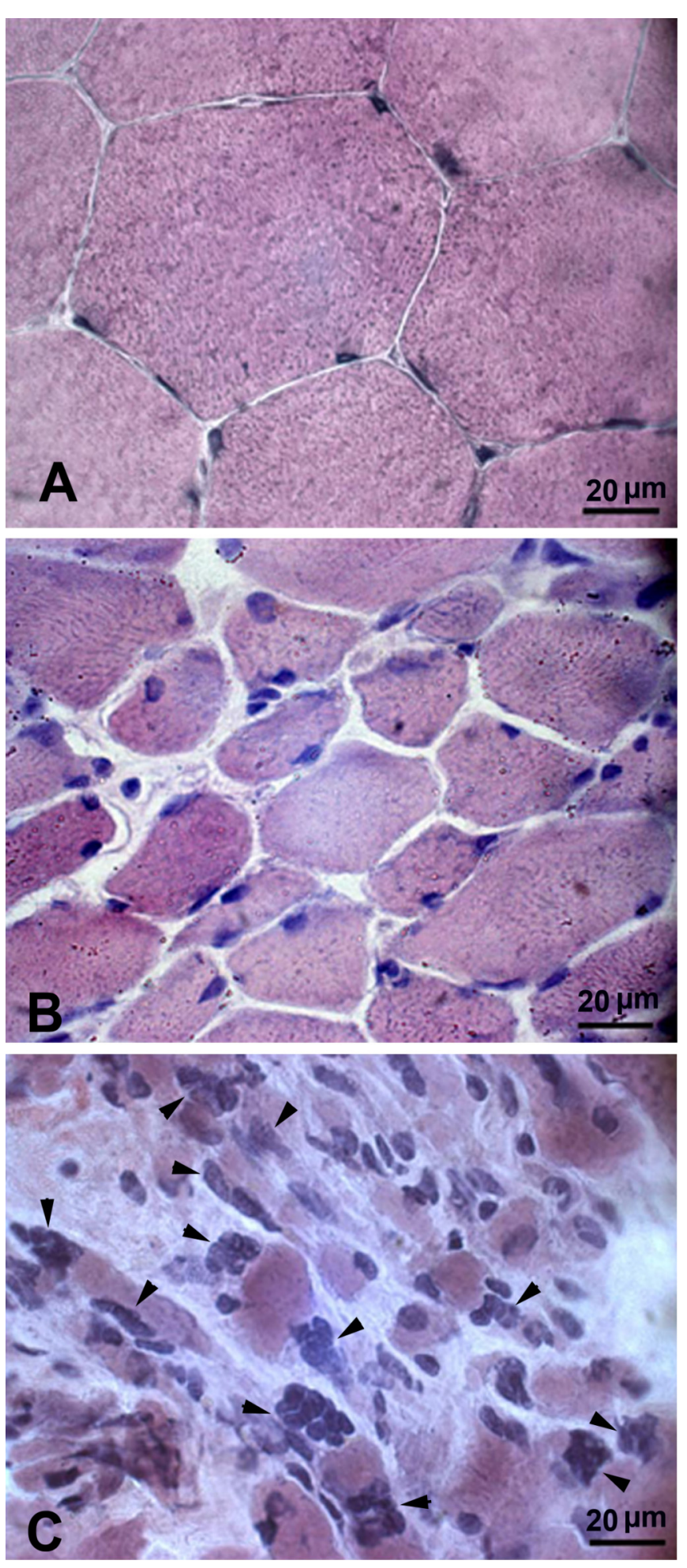

Fig. (1). Severely atrophic muscle fibers with nuclear clumps survive many years in permanently denervated human muscle. Normal and LMN denervated quadriceps muscle. Scale bar: $20 \mu \mathrm{m}$. H\&E stain. (A) Normal muscle. (B) 0,8 year denervated muscle characterized by mild atrophic myofibers, with connective tissue of almost normal appareance. (C) 4,1 year denervated muscle showing severely atrophic myofibers in which the contractile apparatus is almost absent and three or more clumped nuclei (arrowheads) can be identified. The nuclear clumps are better evaluated in oblique muscle fiber sections. 
Table 1. Percentage of Human Severely Atrophic Muscle Fibers with Nuclear Clumps in Muscle Biopsies Harvested from One to Nine Years of Permanent Lower Motoneuron Denervation due to Complete Lumbar-Ischiatic Spinal Cord Injury

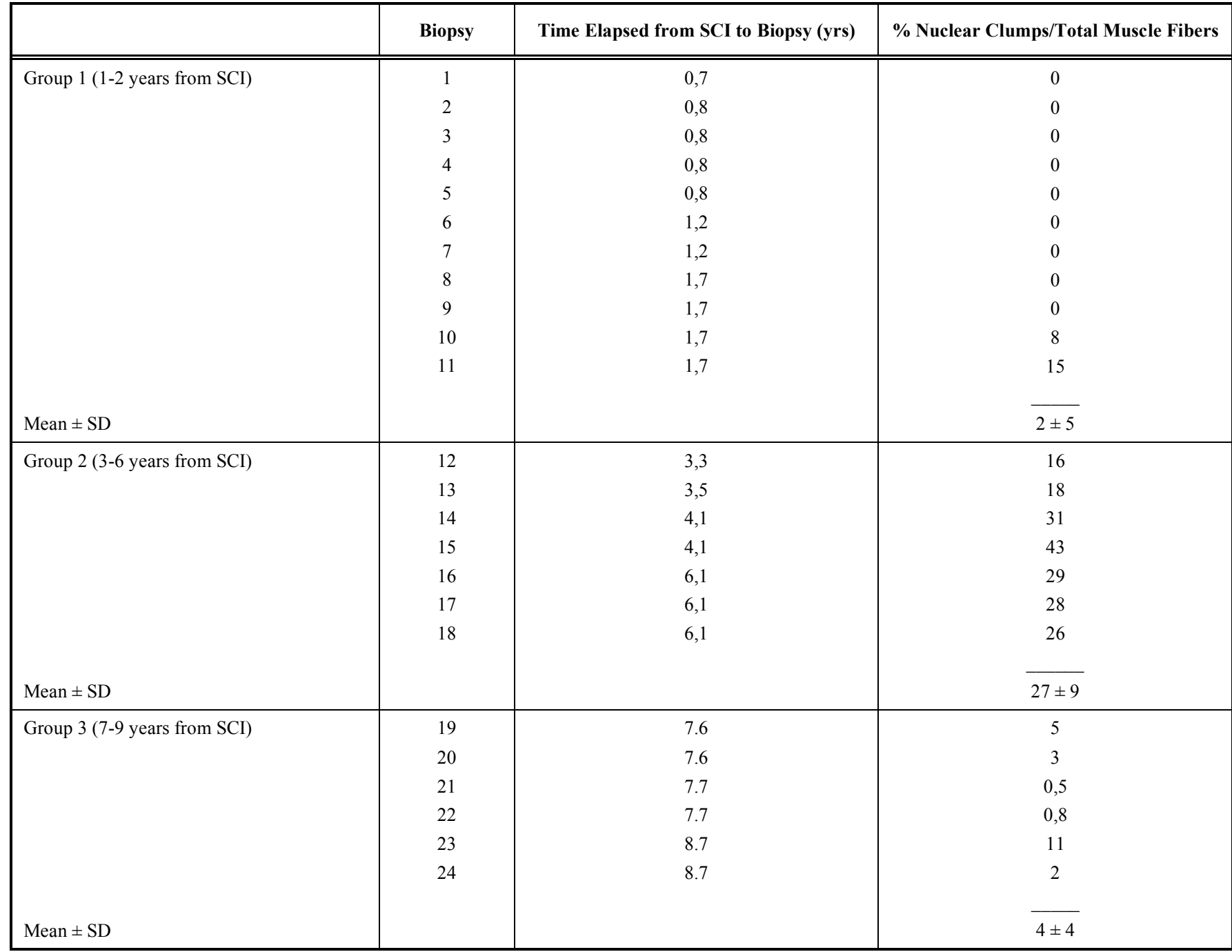

In the muscle sections from biopsies harvested from one to two years after complete denervation ( $\mathrm{n}=11$ ), we observed $2 \pm 5 \%$ of myonuclear clumps (mean $\pm \mathrm{SD}$ ). In biopsies harvested three to five years after spinal cord injury $(\mathrm{n}=7)$, the percentage abruptly and significantly increased to $27 \pm 9 \%(\mathrm{p}<0,001)$. The value significantly decreased in biopsies $(\mathrm{n}=6)$ harvested more than six years from spinal cord injury to $4 \pm 4 \%(\mathrm{p}<0,001$ vs three-six years of denervation).

muscle fibers with nuclear clumps are absent up to 1.5 years of LMN denervation. Typical severely atrophic muscle fibers with nuclear clumps are common in three to six years LMN denervated muscle fibers. In the muscle sections from biopsies harvested from one to two years after complete denervation $(\mathrm{n}=$ 11 ), we observed $2 \pm 5 \%$ of myonuclear clumps (mean $\pm \mathrm{SD}$ ). In biopsies harvested three to five years after spinal cord injury $(\mathrm{n}=7)$, the percentage abruptly and significantly increased to 27 $\pm 9 \%(\mathrm{p}<0,001)$. The value significantly decreased in biopsies $(\mathrm{n}=6)$ harvested more than six years from spinal cord injury to $4 \pm 4 \%$ ( $\mathrm{p}<0,001$ vs three-six years of denervation).

Fig. (2) shows a cryosection from a 5.4-year LMN denervated human muscle biopsy after staining in green with an anti-MHCs antibody. The white arrows point to slow-type green atrophic muscle fibers with centralized nuclei, labeled in blue by Hoechst 33258. The black arrowheads indicates two less atrophic fast type muscle fibers (not stained by the anti-MHCs antibody), which contains several centralized nuclei. These morphological aspects suggest that the severe atrophy with nuclear redistribution is the results of the progressive disorganization of the sarcomeric stuctures of the denervated muscle fibers, which meantime lose the normal coil distribution of myonuclei. Whatever the mechanisms of their rearrangement, the muscle nuclei are for many months (or years) grouped in clusters of tens of nuclei that fill the severely atrophic muscle fibers, being separated by long stretches of amyofibrillar myoplasm.

The size of nuclear clumps is better evaluated in $1 \mu \mathrm{m}$ semi-thin longitudinally sectioned myofibers (Fig. 3). Stretches of 15-20 $\mu \mathrm{m}$ large and 50-100 $\mu \mathrm{m}$ long amyofibrillar sarcoplasm alternates with groups of tens of nuclei that fills 20-30 $\mu \mathrm{m}$ long portions of the fibers. These two $0.3 \mathrm{~mm}$ long portion of severely atrophic muscle fibers contain 10-30 myonuclei, suggesting that loss of nuclei is substantial at these late stage of muscle atrophy (in normal muscle there are 10002000 nuclei per mm of myofiber) $[10,11]$.

\section{DISCUSSION}

In biopsies harvested from patients affected with complete Conus Cauda syndrome (that is, complete and 
permanent lower motor neuron lesion at lumbar-ischiatic level of spinal cord), the muscle tissue, before undergoing adipose and fibrous tissue substitution, progressively shows a severe atrophy of muscle fibers that loses their myofibrillar apparatus and relocates in clumps their nuclei [4]. These features are in sharp contrast with the stable muscle atrophy we described in long-term paraplegics with complete upper motor neuron lesion from three to twenty years of spinal cord injury at thoracic level [12]. Interestingly, we never observed nuclear clumps in this type of disuse atrophy.

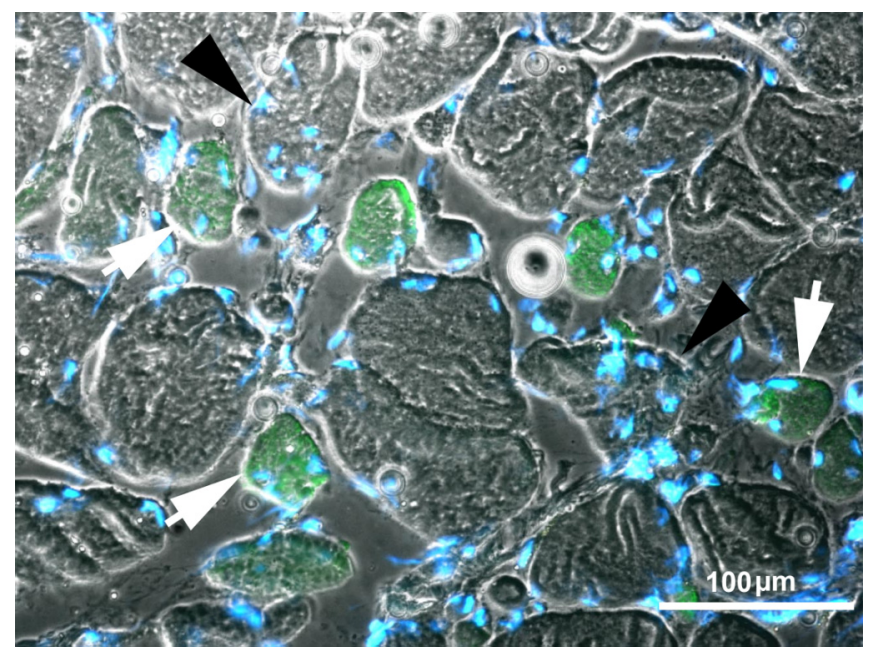

Fig. (2). Immunohistochemical staining with anti-MHCs shows that both fast (larger, not stained muscle fibers pointed by black arrowheads) and the green-labeled slow type muscle fibers (white arrows) present several central nuclei. Scale bar: $100 \mu \mathrm{m}$. from the coil pattern of normal muscle fibers to nuclear clumps.

Up to now, nuclear groupings have been described in muscle biopsies from patients affected with neurodegenerative disorders, like amyotrophic later sclerosis and spinal muscular atrophy $[13,14]$. In those cases, nuclear clumps are rarely present in muscle fibers empty of their sarcomeric structures, because in neurodegenerative diseases the incompleteness of denervation allows reinnervation and thus the reorganization of larger muscle units [14].

\section{CONCLUSION}

Our observations thus confirm that human muscle fibers survive complete and permanent denervation much longer than generally accepted. Nuclear clumps should be considered markers of the long-lasting ability of human muscle fibers to survive to the absence of the nerve. These results provide the rationale to plan research aimed to recover these severely atrophic myofibers, by combining molecular and cellular approaches with functional electrical stimulation that our previous studies shown to restore muscle structure and mass in human long-term denervated and degenerated muscle $[4,7,15-20]$.

\section{ACKNOWLEDGEMENTS}

We thank Mr. V. Gobbo for his valued contribution to the work. This research was supported by EU Commission Shared Cost Project RISE (Contract no. QLG5-CT-200102191), Ludwig Boltzmann Institute of Electrostimulation and Physical Rehabilitation funds, Italian MIUR funds to the Laboratory of Translational Myology, University of Padova,

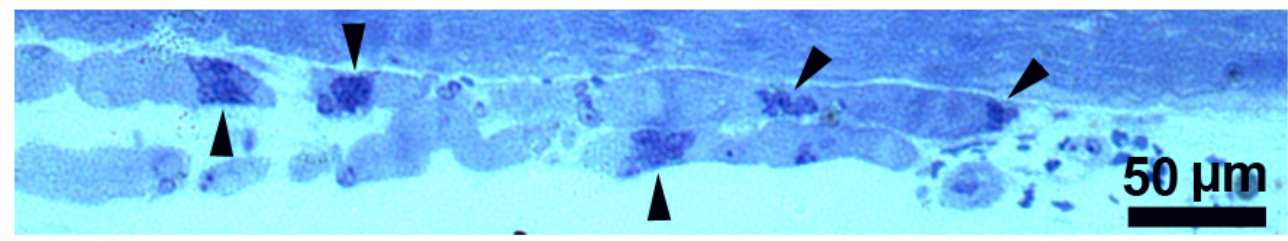

Fig. (3). Longitudinal semi-thin section of severely atrophic myofibers. Arrowheads point to nuclear clumps, which alternates with longer stretches of anucleated, amyofibrillar sarcoplasm. Toludine blu stain. Scale bar: $50 \mu \mathrm{m}$.

Here we show that the severe atrophy characterized by nuclear clumps becomes visible after more than one year from complete and permanent LMN denervation, and that it possibly interests all the surviving muscle fibers between the third and the fifth year of denervation. The immunoistochemical analysis presented in Fig. (2) shows that both fast and slow muscle fibers undergo severe atrophy and nuclear relocation.

The only practical way to perform a quantitative analysis was to count nuclear clumps on muscle cross sections and express the results as percentage of the total muscle fibers identified in each muscle biopsy. Taking into account that after the third year of denervation, approximately $30 \%$ of atrophic myofibers show nuclear clumps (Table 1), and that the clumps of nuclei are separated by longer stretches of amyofibrillar cytoplasm (Fig. 3), we may conclude that almost all the severely atrophic myofibers seen in muscle biopsies from three to five years after complete, permanent lower motor neuron injury, present relocation of their nuclei
Italy, and Italian C.N.R. funds to the Institute of Neuroscience, University of Padova, Italy.
ABBREVIATIONS
$\mathrm{H} \& \mathrm{E}=$ Hematoxilin and eosin
LMN $=$ Lower motor neuron
SCI $=$ Spinal cord injury
$\mathrm{SD}=$ Standard deviation
MHCs $=$ Slow-type myosin heavy chain
TBS $=$ Tris-buffered saline

\section{REFERENCES}

[1] Adami N, Kern H, Mayr W, et al. Permanent denervation of rat Tibialis Anterior after bilateral sciatectomy: Determination of chronaxie by surface electrode stimulation during progression of atrophy up to one year. Basic Appl Myol 2007; 17: 237-43. 
[2] Borisov AB, Dedkov EI, Carlson BM. Interrelations of myogenic response, progressive atrophy of muscle fibres, and cell death in denervated skeletal muscle. Anat Rec 2001; 264: 203-18.

[3] Carlson BM. "The Denervated Muscle" - 45 years later. Basic Appl Myol 2007; 17: 113-7.

[4] Kern H, Boncompagni S, Rossini K, et al. Long-term denervation in humans causes degeneration of both contractile and excitationcontraction coupling apparatus, wich is reversibile by functional electrical stimulation (FES). A role for myofiber regeneration? J Neuropathol Exp Neurol 2004; 63: 919-31.

[5] Biral D, Kern H, Adami N, et al. Atrophy-resistant fibers in permanent peripheral denervation of human skeletal muscle. Neurol Res 2008; 30: 137-44.

[6] Ashley Z, Sutherland H, Lanmuller H, et al. Atrophy, but not necrosis, in rabbit skeletal muscle denervated for periods up to one year. Am J Cell Physiol 2007; 292: 440-51.

[7] Lewis DM, Al-Amood WS, Schmalbruch H. Effects of long-term phasic electrical stimulation on denervated soleus muscle: guinea pig contrasted with rat. J Muscle Res Cell Motil 1997; 18: 573-86.

[8] Kern H, Hofer C, Mayr W, Carraro U. European Project RISE: Partners, protocols, demography. Basic Appl Myol/Eur J Transl Mycol 2009; 19: 211-6.

[9] Rossini K, Zanin ME, Podhorska-Okolow M, Carraro U. To stage and quantify regenerative myogenesis in human long-term permanent denervated muscle. Basic Appl Myol 2002; 12: 277-86.

[10] Aravamudan B, Mantilla CB, Zhan WZ, Sieck GC. Denervation effects on myonuclear domain size of rat diaphragm fibers. J Appl Physiol 2006; 100: 1617-22.
[11] Gundersen K, Bruusgaard JC. Nuclear domains during muscle atrophy: nuclei lost or lost paradigm. J Physiol 2008; 586: 2675-81.

[12] Kern H, Hofer C, Modlin M, et al. Stable muscle atrophy in long term paraplegics with complete upper motor neuron lesion from 3to 20-year SCI. Spinal Cord 2008; 46: 293-304.

[13] Dubowitz V, Sewry CA. Muscle biopsy. A practical approach. Sauders Elsevier Edition 2007. Neurol 2004; 63: 919-31.

[14] Wokke JH. Genes, trials, and care: The dynamics of neuromuscular disease. Lancet Neurol 2004; 3: 1-16.

[15] Boncompagni S, Kern H, Rossini K, et al. Structural differentiation of skeletal muscle fibers in the absence of innervation in humans. Proc Natl Acad Sci USA 2007; 104: 19339-44.

[16] Kern H, Salmons S, Mayr W, Rossini K, Carraro U. Recovery of long-term denervated human muscles induced by electrical stimulation. Muscle Nerve 2005; 31: 98-101.

[17] Kern H, Carraro U. Translational myology focus on clinical Challenges of functional electrical stimulation of denervated muscle. Basic Appl Myol/Eur J Transl Myol 2008; 18: 37-100.

[18] Kern H, Carraro U, Adami N, et al. One year of home-based Functional Electrical Stimulation (FES) in complete lower motor neuron paraplegia: Recovery of tetanic contractility drives the structural improvements of denervated muscle. Neurol Res 2009, in press.

[19] Kern H, Hofer C, Mayr W. Protocols for clinical work package of the European project RISE. Basic Appl Myol/Eur J Transl Myol 2008; 18: 39-44.

[20] Kern H. Funktionelle Elektrostimulation paraplegischer Patienten. Österr Z Phys Med 1995; 5: 1-79.

(C) Kern et al.; Licensee Bentham Open.

This is an open access article licensed under the terms of the Creative Commons Attribution Non-Commercial License (http: //creativecommons.org/licenses/by-nc/ 3.0/) which permits unrestricted, non-commercial use, distribution and reproduction in any medium, provided the work is properly cited. 